\title{
2-D Object Recognition Approach using Wavelet Transform
}

\author{
Kamelsh Kumar $^{1}$ \\ Department of Computer Science \\ Sindh Madressatul Islam University \\ Karachi, Sindh, Pakistan
}

\author{
Riaz Ahmed Shaikh², Rafaqat Hussain Arain ${ }^{3}$, \\ Safdar Ali Shah ${ }^{4}$, Hidayatullah Shaikh ${ }^{5}$ \\ Department of Computer Science \\ Shah Abdul Latif University, Khairpur, Sindh, Pakistan
}

\begin{abstract}
Humans have supernatural ability to observe, analyze, and tell about the layout of the 3D world with the help of their natural visual system. But contrary to machine vision system, it remains a most difficult task to recognize various objects from images being captured by cameras. This paper presents 2-D image object recognition approach using Daubechies (Db10) wavelet transform. Firstly, an edge detection is carried out to delineate objects from the images. Secondly, shape moments have been used for object recognition. For testing purpose, different geometrical shapes such as rectangle, circle, triangle and pattern have been selected for image analysis. Simulation has been performed using MATLAB, and obtained results showed that it accurately identifies the objects. The research goal was to test 2 -D images for object recognition.
\end{abstract}

Keywords-Wavelet transforms; db10; edge detection; object recognition; shape moments

\section{INTRODUCTION}

Since last few years, object recognition has remained one of the challenging tasks in different applications of domain, namely, computer vision, pattern recognition, data mining, object tracking and remote sensing. It can be defined as a process for highlighting, identifying, and extracting the targeted objects from the image and video based repositories. Object recognition is being extensively used in various applications for different purposes, such as in medical technology, researchers are interested in finding the specific regions that can assist doctors to recognize abnormalities in human body, in surveillance and monitoring systems, inspection is necessary security measure to point out a particular person for fraudulent activities. Similarly, in bio related trait systems, individual object verification is required for authentication [1]-[4]. Object recognition is core area of pattern recognition, which uses supervised as well as unsupervised machine learning techniques for mining hidden structures in the data. However, building an efficient object recognition system heavily relies over proper image segmentation method. In this paper, research has been solely carried out for 2-D images for the shape recognition. There are various techniques that have been used for shape representation; however, they are roughly categorized into contour and region methods. First one retrieve the shape from the boundary and second one extracts the shape from the whole region. The comprehensive study on shape features have been given in [5]. The contents of this paper are arranged in the following order. Section one describes the literature review, which is related to 2-D objects for shape recognition and retrieval. Section 2 explains wavelets transform and its methodology for edge detection and object recognition. Section 3 describes the experimental calculation and simulation results for object retrieval and finally Section 4 provides the conclusion.

\section{LITERATURE REVIEW}

Over the past, various approaches have been put forwarded by authors for 2-D images for shape recognition. In [6] JiannDer Lee and Jau-Yien Lee suggested a recognition technique for partial 2-D perspective shapes. This method was able to recognize the unknown shape from arbitrary perspective. However, efficiency of the system was limited for those objects missing only $20 \%$ of data. Similarly, K.C. Wong and Y.Cheng presented a recognition method for polyhedral objects with the aid of triangular pair feature. The rate of identification for objects was relatively high but their method does not considered spatial and temporal information [7]. In other paper [8], P.K Sinha and F-Y Chen, implemented Hough parameter space in which shapes were recognized along with their location. The drawback of this method is that Hough transform is good for line detection but it is not feasible for circle object recognition. The wavelets transform for the 2-D occluded object recognition was given by Tiehua Du and Kah Bin Lim, where boundary of the object was extracted for shape detection. But this method is only suitable for partially overlapped objects [9]. Object corner detection using wavelet transform was proposed by Lu Sun and Y.Y Tang, this method uses local extrema as well as modulus of the transform results for the detection of corner and its arcs. However, the system suffers from few missed corner detections [10]. A linear discriminant analysis LDA technique for box structure objects detection was described by Chia-Chih Chen and J.K Aggarwal [11]. This method outperforms in different angle view of objects but provided some false positive and negative detection results due to over segmentation. Random transform for object matching was suggested by Yi Wan and Ning Wei [12]. This method recognizes the objects from rotated, reflected and scaled projection, while it is only useful for non-occluded objects. Similarly, 2-D objects retrieval and shape matching using B-spline representation was given by Nacera Laiche and Slimane Larabi [13]. A contemporary method of content analysis using shape and spatial layout with MRF has been proposed by Shaikh et al. [14]. Experimental results 
demonstrate the effectiveness of the proposed method to efficient content analysis.

\section{WAVELET TRANSFORM}

Wavelet is a type of frequency transform technique which is being used for signal analysis. Also, it has been an important tool for researchers for image analysis since more than a decade. Wavelet transform is characterized by its orthogonal function, which can be used on limited group of data. It provides multi scale signal analysis using low pass and high pass filter. In this paper, wavelet has been implemented on 2-D images for edge detection and object retrieval. This technique is efficient in the sense that discrete image is decomposed through these filters and change in grey level intensity can easily be detected for object recognition. There are number of wavelets families which are known by their particular name. However, each of them is characterized by its basic scaling function and wavelet shape function; these values are used for particular signal analysis.

\section{A. Proposed Methodology}

Our proposed methodology is based on our previous research work which was carried out for 2-D edge detection [15]. In this paper, it has been extended for 2-D object recognition.

Initially, an original image in Fig. 1 is given as an input to db10 wavelet transform for edge detection [16]. After that it decomposes image up to three levels for separation of approximation and details co-efficient. The details contain higher frequencies which correspond to the actual edges, so we have suppressed the approximation effect.

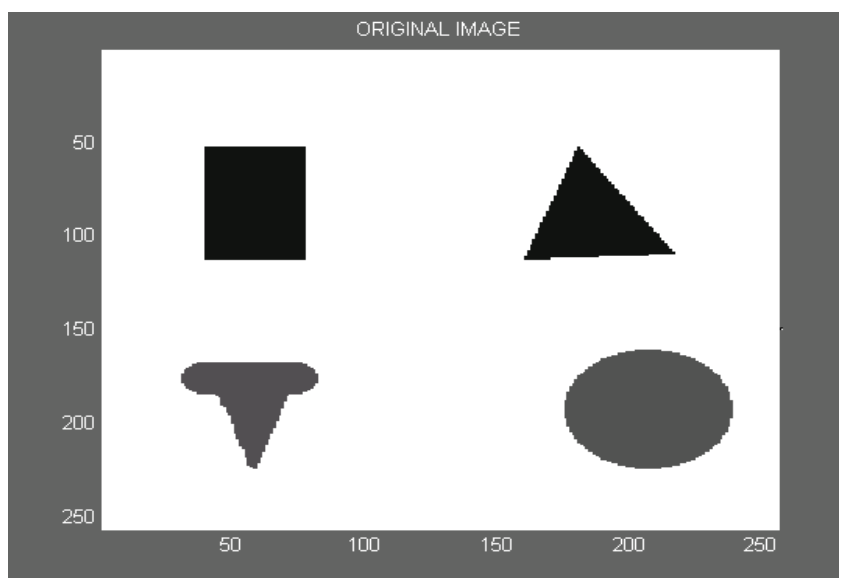

Fig. 1. Original image.

Secondly, an original image has been reconstructed for the detection of image edges as shown in Fig. 2. For this purpose, an algorithm is devised below:

\section{Algorithm for Reconstruction of Image \\ 1. Take approximations as well as detailed coefficients for three level decomposition.}

2. Perform up sampling.

3. Use low and high pass filter (Reconstructed filter).
4. Save them in an array.

5. Combine them to get approximations and details.

6. Save them into workspace.

7. Now, this is first level reconstruction.

8. Do it again from step 2 through 6 by taking first level reconstructed approximations and details.

9. This process is carried out until we get original image.

10. The edges of original image will be retrieved at $\mathrm{CA} 3=0$

11. Meanwhile, Use thresholding to nullify ghost edges.

12. Finally, an original image will be displayed with edges.

\section{B. Object Recognition}

Once the edges are found, the next step is to recognize objects. For this purpose, database has been created as shown in Fig. 3, which consists of different object patterns, such as rectangle, triangle, circle and pattern. Later, these objects have been taken as query image for object recognition.

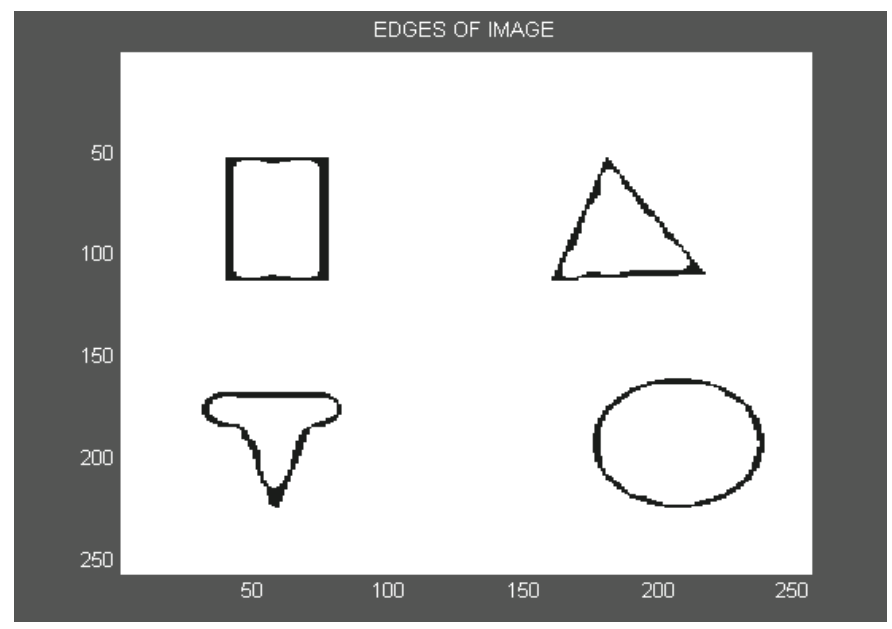

Fig. 2. Edges of the image.
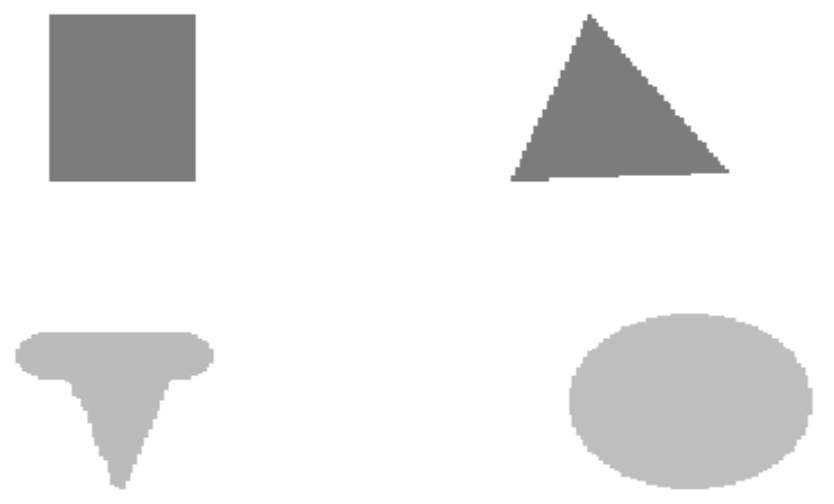

Fig. 3. Database for object recognition. 
The algorithm for object recognition has been described as below.

\section{Algorithm for Object Recognition}

1. Find first pixel

2. Store its location in arrays individually containing rows and columns

3. Zero the pixel from an input image

4. Check its 8 neighbour-hood connectivity

5. Save its neighbour-hood pixel locations in the same array containing first pixel location

6. Simultaneously, zeroing those pixels from input image

7. Taking pixels immediately next to the first pixel from arrays containing locations of detected pixels

8. Check its 8 neighbour-hood connectivity

9. Store the location of the detected pixel in the same array declared previously

10. This process is repeated until the time 8 neighbour-hood connectivity pixels of preceding pixels exit

11. Declare it the first object

12. Again first pixel is detected

13. Repeat steps from 2 to 11

14. Declare it the second object

\section{Shape Moments}

Shape moments are good at exploiting global features of an image which is useful for object recognition. These moments are defined over Cartesian moments that contain basic set function $\{x p, y q\}$. However, $(p+q)$ th $2-D$ geometric moments for an image I (x,y) are denoted by a matrix mpq and can be narrated as:

$$
m_{p q}=\int_{-\infty}^{\infty} \int_{-\infty}^{\infty} x^{p} y^{p} I(x, y) d x d y
$$

In (1) $\mathrm{I}(\mathrm{x}, \mathrm{y})$ depicts the continuous image function, which is stored as two- dimensional array $\mathrm{I}(\mathrm{i}, \mathrm{j})$ in the computer memory. The $\mathrm{i}=0,1 \ldots, \mathrm{Nx}-1$ and $\mathrm{j}=0,1, \ldots, \mathrm{Ny}-1$ represents an array $\mathrm{Nx} \times \mathrm{Ny}$. However, moments are calculated by omitting integrals with summation as shown in (2).

$$
m_{p q}=\sum_{i} \sum_{j} I(i, j) \cdot i^{p} j^{q}
$$

And, also normalized moments for $\mathrm{x}-\mathrm{y}$ axis can be approximated by:

$$
m_{p q}=\sum_{i} I\left(x_{i}, y_{i}\right) \cdot x_{i}^{p} y_{i}^{q}
$$

Similarly, in (3) summation is performed for all image pixels where xi, yi are central coordinates of ith pixel. Shape moments are efficient in finding intensity distribution of an image. These are invariant to translation, rotation and scale. Therefore, central moments, normalized moments and invariant moments have been calculated for each object and then stored in an array. Later, individual query object is recognized through comparing its actual moments in the database.

\section{Mathematical Calculation}

In this section, central moments, normalized moments and invariant moments of Rectangle, Triangle, Pattern and Circle have been calculated for shape recognition, respectively as shown below in Table I.

In addition to aforementioned tabular calculation, central, normalized and invariant moments have been computed using following formulae:

1) Central moments

$\mathrm{r}-\mathrm{bar}=\operatorname{mean}(\mathrm{r})$;

$\mathrm{c}-\mathrm{bar}=\operatorname{mean}(\mathrm{c})$;

Momlist (i) $=(\mathrm{r}(\mathrm{i})-$ rbar $) \wedge \mathrm{p} *(\mathrm{c}(\mathrm{i})-\mathrm{cbar}) \wedge \mathrm{q}$;

Momlist $(i)={ }_{\left(\left(r_{i}\right)-\bar{r}\right)} p *\left(c_{i}-\bar{c}\right) q$;

Central moments $(\mathrm{CM})=$ sum (momlist);

Where, $\mathrm{p}$ and $\mathrm{q}$ are showing the order of $\mathrm{CM}$, are saved in an array.

\section{2) Normalized moments}

\section{$\mathrm{NCM}=\mathrm{CMpq} / \mathrm{CM} 00^{\wedge} \mathrm{J}$}

Whereas $\mathrm{J}=(\mathrm{p}+\mathrm{q}) / 2+1$;

Normalized moments are saved in an array.

3) Invariant moments

Inva_moment $1=\mathrm{h} 20^{\wedge} 2+\mathrm{h} 02^{\wedge} 2$;

Inva_moment $2=(\mathrm{h} 20-\mathrm{h} 02)^{\wedge} 2+4 * \mathrm{~h} 11^{\wedge} 2$;

Inva_moment $3=(\mathrm{h} 30-3 * \mathrm{~h} 12)^{\wedge} 2+(\mathrm{h} 03-3 * \mathrm{~h} 21)^{\wedge} 2$;

Inva_moment $4=(\mathrm{h} 30+\mathrm{h} 12) \wedge 2+(\mathrm{h} 03+\mathrm{h} 21)^{\wedge} 2$;

Inva_moment5 $=(\mathrm{h} 303 * \mathrm{~h} 12) *(\mathrm{~h} 30+\mathrm{h} 12) *((\mathrm{~h} 30+\mathrm{h} 12)$

$\wedge 2-3 *(\mathrm{~h} 03+\mathrm{h} 21)^{\wedge} 2+(3 * \mathrm{~h} 21+\mathrm{h} 03) *(\mathrm{~h} 03+\mathrm{h} 21)$

$*\left(3 *(\mathrm{~h} 30+\mathrm{h} 21)^{\wedge} 2-(\mathrm{h} 03+\mathrm{h} 21)^{\wedge} 2\right)$;

Inva_moment6 $=(\mathrm{h} 20-\mathrm{h} 02) *((\mathrm{~h} 30+\mathrm{h} 21) \wedge 2-(\mathrm{h} 03+\mathrm{h} 21) \wedge 2)+$ $4 * \mathrm{~h} 11 *(\mathrm{~h} 30+\mathrm{h} 12) *(\mathrm{~h} 03+\mathrm{h} 21)$;

Inva_moment $7=(3 * \mathrm{~h} 21-\mathrm{h} 03) *(\mathrm{~h} 30+\mathrm{h} 12) *(\mathrm{~h} 30+\mathrm{h} 12)$

$\left.\wedge 2-3 *(\mathrm{~h} 03+\mathrm{h} 21)^{\wedge} 2\right)-(\mathrm{h} 03-3 * \mathrm{~h} 21) *(\mathrm{~h} 03+\mathrm{h} 21) *(3 *$

$\left.(\mathrm{h} 30+\mathrm{h} 12)^{\wedge} 2-(\mathrm{h} 03+\mathrm{h} 21)^{\wedge} 2\right)$;

Invariant moments are saved.

TABLE I. SHAPE MOMENTS CALCULATION

\begin{tabular}{|l|lllllllll|}
\hline $\begin{array}{l}\text { Moment } \\
\text { Triangle }\end{array}$ & 0.0197 & 0.0014 & 0.0047 & 0.0000 & 0.0000 & 0.0000 \\
\hline $\begin{array}{l}\text { Moment } \\
\text { Circle }\end{array}$ & 0.0000 & 0.0127 & 0 & 0 & 0 & 0 & 0 & 0 & \\
\hline $\begin{array}{l}\text { Moment } \\
\text { Rectangle }\end{array}$ & 0.0201 & 0.0062 & 0 & 0 & 0 & 0 & 0 & \\
\hline $\begin{array}{l}\text { Moment } \\
\text { Pattern }\end{array}$ & 0.0254 & 0.0017 & 0.0094 & 0.0004 & 0.0000 & 0.0001 \\
\hline
\end{tabular}




\section{RESUlTS AND DISCUSSIONS}

In this section, we have shown results which are being generated using MATLAB programming. For this purpose, discussion has been made for object detection. In order to detect object, an input query image is feed to a system, after that it computes its features using shape moments. Then matching of features is achieved from the database. And finally system recognizes shape of an object. It can be observed from Fig. 4 to 7 that detected object are rectangle, triangle, pattern, and circle respectively. From these results, it can be concluded that wavelet transform for edge detection and shape moments for object recognition performed well over grey level intensity images.

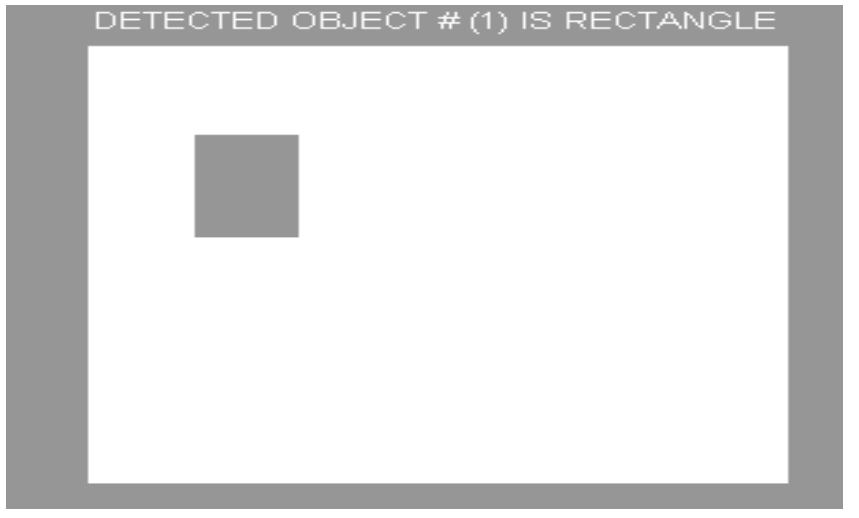

Fig. 4. Rectangle object detection.

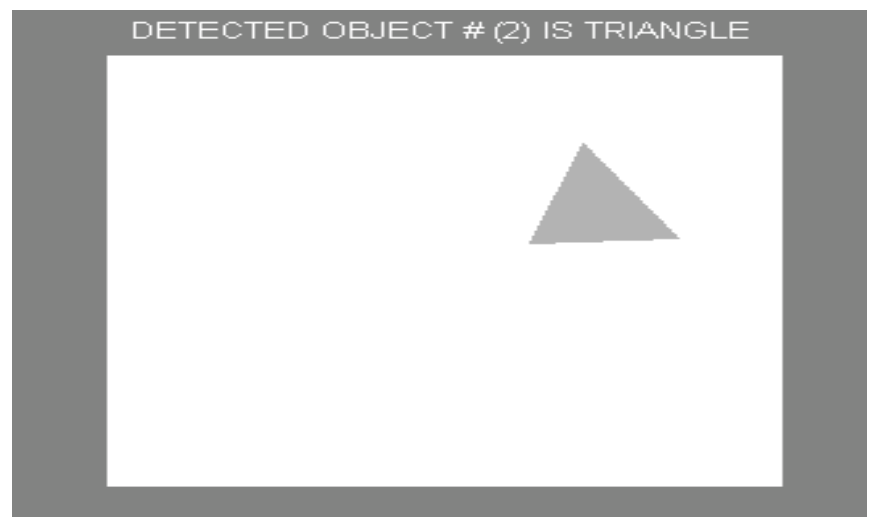

Fig. 5. Triangle object detection.

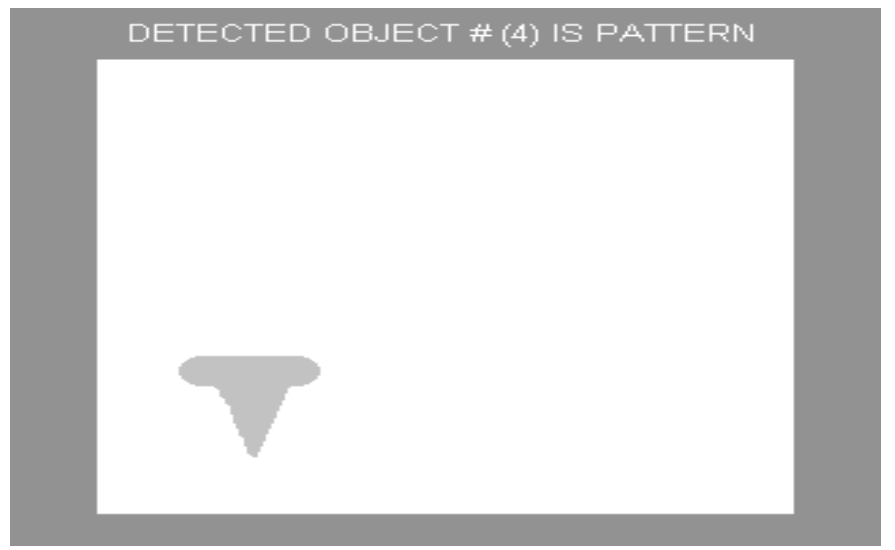

Fig. 6. Pattern object detection.

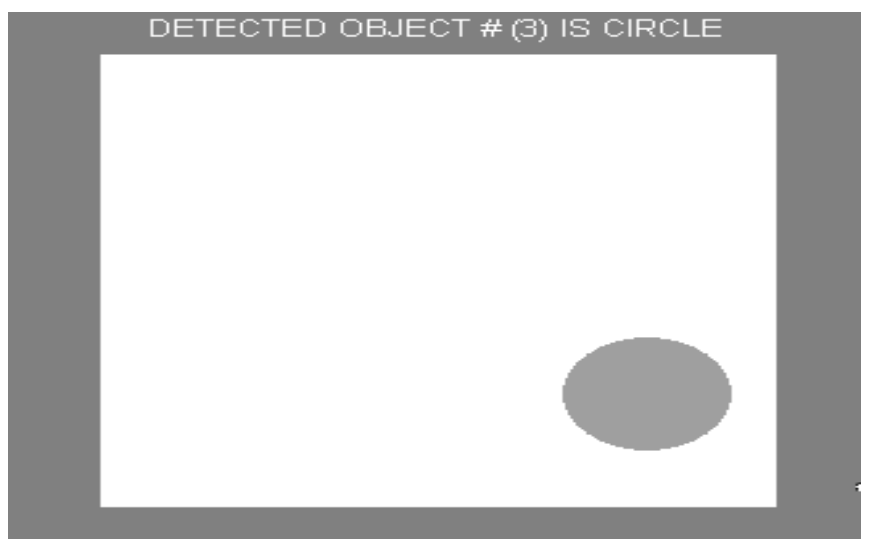

Fig. 7. Circle object detection.

\section{CONCLUSIONS}

In this paper, 2-D image edge detection and object recognition approach was achieved using Db10 wavelet transform and with shape moments. Simulation results showed that wavelet transforms for edge detection and shape moments for object recognition generate efficient results for 2-D type images. And it is suitable for grey scale images for object recognition. However, proposed system has some limitation, as it does not work well with colour images. Therefore, in future one can improve these algorithms for the sake of high level pattern recognition.

\section{REFERENCES}

[1] Patrick Connor, Arun Ross, "Biometric Recognition By Gait: A Survey of Modalities and Features", Journal Of Computer Vision and Image Understanding, vol. 167, pp. 1-27, 2018.

[2] K. Nguyen, C. Fookes, R. Jillela, S. Sridharan, A. Ross, "Long Iris Recognition: A Survey", Journal of Pattern Recognition, vol. 72, pp. 123-143, 2017.

[3] Swaroop Guntupalli, M. Ida Gobbini, "Reading Faces: From Features to Recognition", Journal of Trends in Cognitive Sciences, vol. 21, no. 12, pp. 915 916, 2017.

[4] J. Liang, J. Zhou, L. Tong, X.Bai, B.Wang, Material Based Silent Object Detection from Hyper spectral Images, Journal of Pattern Recognition, vol.76, pp. 476-490, 2018.

[5] Dengsheng Zhang, Guojun Lu, "Review of Shape Representation and Description Techniques", The Journal of Pattern Recognition Society, vol. 34, pp. 1-19, 2004.

[6] Jiann-Der Lee, Jau-Yien Lee and Chin-Hsing Chen, "A New Recognition Method for Partial 2-D perspective", IEEE Region 10 Conference on Computer and Communication System, pp. 601-605, September 1990.

[7] K.C. Wong, Y. Cheng and J. Kittler, “ Recognition of Polyhedral Objects Using Triangle Pair Features, IEEE Proceedings-1, vol. 140, pp. 72- 85, February, 1993.

[8] P. K. Sinha, F-Y Chen and R E N Horne, "Recognition and Location of Shapes in the Hough Parameter Space", IEE, Savoy Place, London WC2R 0BL, UK, 1993.

[9] Tiehua Du, Kah Bin Lim, and Geok Soon Hong, "2D Occluded Object Recognition Using Wavelets", International Conference on Computer and Information Technology, vol. 4, 2004.

[10] Lu Sun, Y. Y Tang and Xinge You, "Corner Detection for Object Recognition by Using Wavelet Transform", International Conference on Machine Learning and Cybernetics, pp. 4347-4351, August. 2004.

[11] Chia-Chih Chen and J.K Aggarwal, "Recognition of Box-Like Objects by Fusing Cues of Shapes and Edges", IEEE, 2008. 
[12] Yi Wan, and Ning Wei, “A fast Algorithm for Recognizing Translated, Rotated, Reflected, and Scaled Objects from only their projections", IEEE Signal Processing Letters, vol. 17, pp. 71-74, January. 2010.

[13] NaceraLaiche, SlimaneLarabi, "Retrieval of 2-D Objects and Shape Matching Using B-splines Representation", International Conference on Signal and Image Processing Applications", pp. 495-500, 2011.

[14] Riaz Ahmed Shaikh, Jian-Ping Li, Asif Khan, Kamlesh Kumar, "Content Analysis Using Shape and Spatial Layout with Markov Random Field", Indian Journal of Science and Technology, Vol 9(7), pp. 1-6, February 2016.

[15] Kamlesh Kumar, Jian-Ping Li, Saeed Ahmed Khan, "Image Edge Detection Scheme Using Wavelet Transform", International Conference on Wavelets Active Media and Information Processing, vol. 11, pp. 261256, December. 2014.

[16] DAUBECHIES, Ingrid. Ten Lectures on Wavelets. Philadelphia, Pennsylvania: Society for Industrial and Applied Mathematics, 1992. CBMS-NSF regional conference series in applied mathematics; vol. 61.ISBN 0898712742. 